\title{
Methylprednisolone Acetate Versus Dexamethasone Injection for Trigger Finger: A Double-blind Randomized Controlled Trial
}

\author{
Porames Suwanno, M.D., Chaiwat Chuaychoosakoon, M.D.
}

Department of Orthopedics, Faculty of Medicine, Prince of Songkla University, Hat Yai, Songkhla 90110, Thailand. Received 19 April 2020 • Revised 15 October 2020 • Accepted 20 October 2020 • Published online 30 March 2021

\begin{abstract}
:
Objective: The study investigated the resolution of triggering by either a soluble methylprednisolone acetate or dexamethasone injection for idiopathic trigger finger at 24-week after injection.

Material and Methods: One hundred and six patients were enrolled in a prospective randomized controlled trial (53 methylprednisolone acetate arms, 53 dexamethasone arms). The outcome was evaluated at 2, 6, 12 and 24-week follow-up. Results: The absence of triggering was documented in 32 of 43, 36 of 41 patients in the methylprednisolone cohort and in 20 of 43, 29 of 32 patients in the dexamethasone cohort at 6 and 24-week after injection. The methylprednisolone acetate cohort had significantly better satisfaction on a visual analog scale, tenderness, and locking at 2-week follow-up, and had significantly better satisfaction on resolution of triggering, tenderness, snapping, locking and the Disabilities of the Arm, Shoulder and Hand score at 6-week follow-up compared to those in the dexamethasone cohort.
\end{abstract}

Conclusion: Methylprednisolone acetate is better in short term clinical outcomes than dexamethasone

Keywords: dexamethasone, injection, methylprednisolone acetate, trigger finger

\section{Contact: Chaiwat Chuaychoosakoon, M.D.}

Department of Orthopedics, Faculty of Medicine, Prince of Songkla University,

Hat Yai, Songkhla 90110, Thailand.

E-mail: psu.chaiwat@gmail.com, chaiwat.c@psu.ac.th

This is an open access article under the CC BY-NC-ND license

(http://www.jhsmr.org/index.php/jhsmr/about/editorialPolicies\#openAccessPolicy).

J Health Sci Med Res 2021;39(5):381-390 doi: 10.31584 /jhsmr.2021794 www.jhsmr.org 


\section{Introduction}

Trigger finger is common chief complaint in orthopaedic clinics. It arises because of an inflammation and constriction of the A1 pulley, which leads to pinch the flexor tendon of the finger. There are various treatments for trigger finger including non-operative and operative treatments. For non-operative treatment, corticosteroid injection is a commonly used treatment in case of trigger finger. Several studies showed the effective treatment of corticosteroid over placebo treatment. ${ }^{1-5}$ The efficacy of treatment is relatively high in all of corticosteroid group but it also has the high recurrence rate. 6 The successful rate treatment of corticosteroid is between $47.0 \%$ and $86.0 \%{ }^{1-3,5-9}$, while the recurrence rate of corticosteroid was between $4.5 \%$ and $29.0 \%$. ${ }^{6}$ The lowest recurrence rate of corticosteroid was dexamethasone that was $4.5 \%$ at 1 -year follow-up. ${ }^{8}$

Ring et al. ${ }^{6}$ reported that the efficacy of triamcinolone acetate [10 milligram per milliliter $(\mathrm{mg} / \mathrm{mL})] 1 \mathrm{~mL}$ for treatment trigger finger was $48.7 \%$ at $12-$ week follow-up. At high dose of triamcinolone acetate $(40 \mathrm{mg} / \mathrm{mL}, 0.5 \mathrm{~mL})$, the success rate was increased up to $70.0 \%$. Therefore, higher dose of triamcinolone acetate showed a greater capacity to recover the symptoms of trigger finger.

The volume of corticosteroid injection in trigger finger was usually at $0.5-1 \mathrm{~mL}$. The efficacy of these volume was approximately 47.0-70.0\%. However, increased volume of corticosteroid improved the efficacy of trigger finger treatment. Sato et al. ${ }^{8}$ reported that high volume of methylprednisolone acetate $(40 \mathrm{mg} / \mathrm{mL}) 2 \mathrm{~mL}$ showed the efficacy of $86.0 \%$ at $24-$ week follow-up.

The hypothesis of this study is that high concentration and volume of corticosteroid may increase the efficacy of corticosteroid for trigger finger treatment. According to previous studies, there was few reports on the efficacy of dexamethasone. This study, therefore, investigated the effect of common use corticosteroid (methylprednisolone acetate) and less common use corticosteroid (dexamethasone) on trigger finger treatment.

\section{Material and Methods}

The method was a double-blind randomized controlled trial, conducted at the hospital between December, 2014 and January, 2016. This study was approved by our Institutional Ethics Committee (Project Number: REC 57053-11-1). The inclusion criteria was patient (greater than 18 year-old) with an idiopathic trigger finger classified as Quinnell grade 1-4. The exclusion criteria was inflammatory joint disease or autoimmune arthritis, multiple digits involved, history of tendon injury of the same digit, a pregnancy or suspected pregnancy, current breast feeding, local infection, history of steroid use in oral or injected form, giant cell of tendon sheath in the same digit, history of steroid allergy, bleeding tendency, and use of anti-coagulopathy drugs. The primary outcome was an absence of finger symptoms. The secondary outcomes were the recurrence of trigger finger, a visual analog scale, tenderness, snapping, locking, the Disabilities of the Arm, Shoulder and Hand (DASH) score and tip to palm distance $(\mathrm{mm})$ and complications. Tenderness was evaluated by the palpation at A1 pulley. The positive result was recorded if the patient had pain on palpation.

Informed consent were obtained from all patients prior the experiment. One hundred and six patients were enrolled and randomized for intra-tendon sheath injection with either methylprednisolone acetate $40 \mathrm{mg} / \mathrm{mL}(53$ patients) or dexamethasone $4 \mathrm{mg} / \mathrm{mL}$ (53 patients), according to a sequence determined by an envelope in a series of blocks of four (Figure 1). 


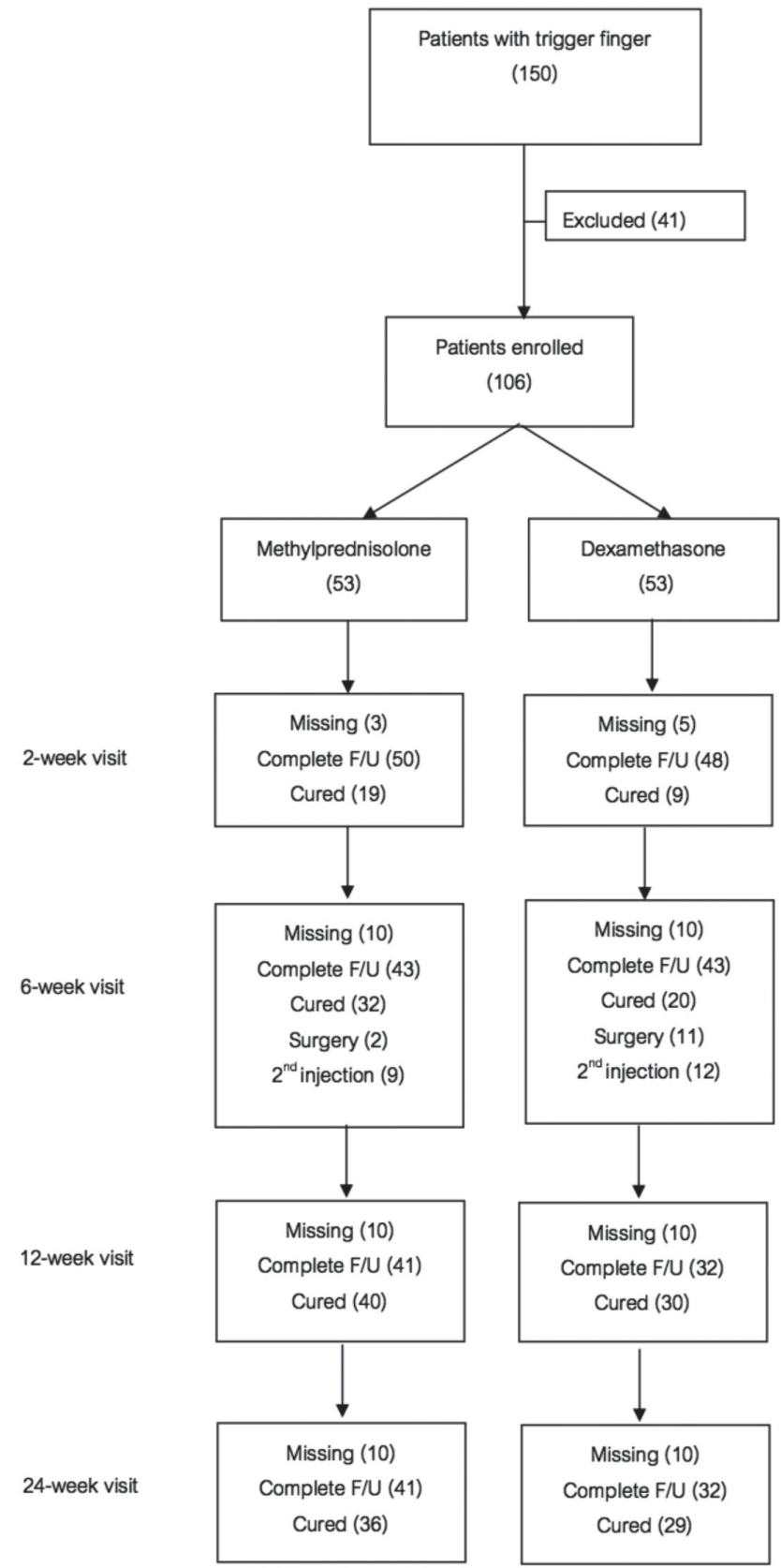

$F N=$ follow - up

Figure 1 Flowchart of patient disposition (follow-up)

During the injection, the patient's eyes were covered with an eye patch. A mixed solution for injection composed of $2 \mathrm{~mL}$ soluble corticosteroid solution and $0.5 \mathrm{~mL} 1.0 \%$ lidocaine without adrenaline. A 27-gauge needle was used for steroid injection. The patient's hand was prepared with povidone-iodine and the digits were slightly extended. The location of the metacarpal head was palpated, then introduced the needle through the skin, subcutaneous, directly into the A1pulley and underneath the flexor tendon (Figure 2). Light pressure was applied to the plunger while simultaneously withdrawing the needle slowly in order to locate the space between tendon and A1 pulley. When the needle tip emerged from the tendon substance, dramatic relief of resistance was felt, then injected the mixed solution for $2 \mathrm{~mL}$. After the injection, the site of injection was applied with cold pack for 5 minutes and the patient was allowed to move the finger freely.

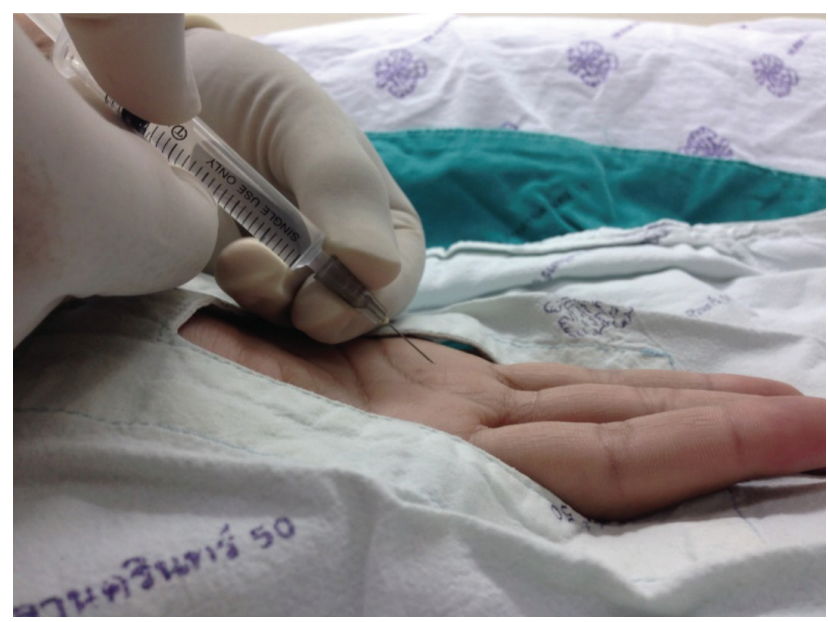

Figure 2 Steroid injection at A1 pulley

The follow-up appointment was made at 2, 6, 12 and 24-weeks after the injection. By providing blinded treatment information, the independent orthopaedist evaluated the patients for Quinnell grading, a visual analog scale, tenderness, snapping, locking, DASH score and tip to palm distance and complications. At 6-week follow-up, the patients who had the remaining symptoms of trigger finger were offered a treatment either a second injection 
of corticosteroid or percutaneous A1 release surgery. The patients made the decision based on the advantages and disadvantages of each treatments given by the orthopaedist. In the case of percutaneous A1 pulley release, the patients were excluded from this study at 12 and 24-week followup.

The primary objective was to compare the efficacy of corticosteroid injection for trigger finger between methylprednisolone acetate and dexamethasone. The secondary objective was to compare recurrence rates, satisfaction on a visual analog scale, tenderness, snapping, locking, the DASH score and tip to palm distance $(\mathrm{mm})$ at 2, 6, 12 and 24-week follow-up.

At the time of enrollment, all patients completed the DASH questionnaire including a demographic data (age, gender, occupation, Body mass index, underlying disease, dominant hand, the involved fingers, duration of symptom, a visual analog scale, snapping, locking and tip to palm distance). After completing the questionnaire, each patient was examined by a hand surgeon, and the trigger finger was graded according to the Quinnell system.

Subjects were reexaminaed at 2, 6, 12 and 24-week after their initial corticosteroid injection. At each time of follow-up, the symptoms of each patient was re-evaluated according to the Quinnell grade, and the patient completed the DASH questionnaire, a visual analog scale, snapping, locking and tip to palm distance. For the visual analogue scale, the patients were asked to make a mark on a line between 0 and 10. Any trigger finger with a Quinnell grade 0 was rated as cured, Quinnell greater than 0 was considered unresolved, and trigger finger which changed from a Quinnell grade 0 was rated as recurrent

Demographic and clinical data were collected using a standard record form. The data were inserted into a computer spreadsheet. Sample size was calculated based on a two-sided a of 0.05 , a statistical power of 0.90 . The sample sizes were 41 patients for each cohort group. The result was analyzed according to the intention to treat principle. Statistical analysis was conducted to produce descriptive statistics for each treatment group. Continuous variable was assessed using the student T-test in normal distribution and Mann-Whitney $U$ test in non-normal distribution. Dichotomous and categorical variables were used Mann-Whitney $U$ test. Change in symptoms (Quinnell grade, DASH score, a visual analog scale, snapping, locking and tip to palm distance) between treatment groups were assessed using the Wilcoxon-signed rank test. The statistical significance was accepted at a probability value of 0.05. Data were analyzed using R program and "epicalc" package (version 3.4.3; R Foundation for Statistical Computing, Vienna, Austria).

\section{Results}

One hundred and six patients with either methylprednisolone acetate (53 patients) or dexamethasone (53 patients) completed at 2, 6, 12 and 24-week follow-up.

\section{Baseline patient characteristics}

There were no statistical differences in demographic data between the methylprednisolone acetate and dexamethasone cohorts (Table 1). The average duration of symptoms was 8-weeks in the methylprednisolone acetate cohort and 12-weeks in the dexamethasone cohort ( $p$-value 0.25). The baseline DASH score averaged 71 points in the methylprednisolone acetate cohort and 70 points in the dexamethasone cohort. The initial Quinnell grades were as follows: 59 patients (32 in the methylprednisolone acetate cohort and 27 in the dexamethasone cohort) were rated grade 3; 35 were rated grade 2 (15 in the methylprednisolone acetate cohort and 20 in the dexamethasone cohort), and 12 were rated grade 1 ( 6 in each cohort) ( $p$-value=0.636). 
Table 1 Demographics

\begin{tabular}{|c|c|c|c|}
\hline Factors & $\begin{array}{l}\text { Methylprednisolone } \\
(n=53)\end{array}$ & $\begin{array}{l}\text { Dexamethasone } \\
(n=53)\end{array}$ & $p$-value \\
\hline Age (years) & $56(52.0,59.5)$ & $56(6.2)$ & 0.398 \\
\hline Sex & & & 0.249 \\
\hline Male & 27 (50.9) & $24(45.3)$ & \\
\hline Female & $26(49.1)$ & $29(54.7)$ & \\
\hline BMI $\left(\mathrm{kg} / \mathrm{m}^{2}\right)$ & $23.6(22.1,25.4)$ & $22.1(21.1,24.1)$ & $0.015^{*}$ \\
\hline \multicolumn{4}{|l|}{ Underlying disease } \\
\hline Diabetes mellitus & $5(9.4)$ & $5(9.4)$ & $>0.05$ \\
\hline Hypertension & $7(13.2)$ & $8(15.1)$ & $>0.05$ \\
\hline Dyslipidemia & $10(18.9)$ & $12(22.6)$ & 0.791 \\
\hline Dominant hand & & & 0.494 \\
\hline Left & $3(5.7)$ & $0(0.0)$ & \\
\hline Right & $50(94.3)$ & $53(100.0)$ & \\
\hline Finger & & & 0.226 \\
\hline Thumb & $17(32.1)$ & $16(30.2)$ & \\
\hline Index & $10(18.9)$ & $3(5.7)$ & \\
\hline Long & $17(32.1)$ & $19(35.8)$ & \\
\hline Ring & $9(16.9)$ & $14(26.4)$ & \\
\hline Little finger & $0(0.0)$ & $1(1.9)$ & \\
\hline Duration (weeks) & $8(4.0,19.0)$ & $12(4.0,24.0)$ & 0.250 \\
\hline Symptomatic hand & & & $>0.05$ \\
\hline Left & $20(37.7)$ & $18(33.9)$ & \\
\hline Right & $33(62.3)$ & $35(66.1)$ & \\
\hline VAS & $5(4.0,7.0)$ & $6(5.0,8.0)$ & 0.099 \\
\hline
\end{tabular}

*Statistical significance $\mathrm{p}-$ value $<0.05$

$\mathrm{BMI}=$ body mass index, $\mathrm{kg} / \mathrm{m}^{2}=$ kilogram per square meter, VAS=visual analog scale

\section{The successful treatment after $1^{\text {st }}$ injection}

The resolution of trigger finger after $1^{\text {st }}$ time steroid injection in the methylprednisolone acetate cohort was 19 in 50, 32 in 43 at 2 and 6-week follow-up; in the dexamethasone cohort it was 9 in 48, 20 in 43 at 2 and 6 -week follow-up. The change in resolution of trigger finger at 6-week follow-up was significantly different between the methylprednisolone acetate cohort and the dexamethasone cohort $(p-$ value $=0.015)$, and no significant difference was found between the methylprednisolone acetate and dexamethasone groups at 2-week follow-up.

\section{Resolution of trigger finger}

The resolution of trigger finger after steroid injection in the methylprednisolone acetate cohort was 19 in 50, 32 in 43, 40 in 41, and 36 in 41 at 2, 6, 12 and 24-week follow-up; in the dexamethasone cohort it was 9 in 48 , 20 in 43, 30 in 32, and 29 in 32 at 2, 6, 12 and 24-week follow-up. The change in resolution of trigger finger at 6-week follow-up was significantly different between the methylprednisolone acetate cohort and the dexamethasone cohort ( $p$-value $=0.015$ ), and no significant difference was found between the 2 groups at 2, 12 and 24-week followup. 


\section{The overall change in Quinnell grading}

The overall change in Quinnell grading was shown in Figure 3. The mean grade improvement for both cohorts was shown in Figure 1. The mean grade in the methylprednisolone acetate cohort was 2, 0, 0, 0 at 2, 6, 12 and 24-week follow-up. The mean grade in the dexamethasone cohort was 2, 1, 0, 0 at 2, 6, 12 and 24-week follow-up. The change in grading at 6-week follow-up was significantly different between the methylprednisolone acetate cohort and the dexamethasone cohort ( $p$-value $=0.003$ ).

\section{The satisfaction on a visual analog scale}

The satisfaction on a visual analog scale was shown in Figure 4. The initial satisfaction on a visual analog scale was 5 and 6 in the methylprednisolone acetate cohort and in the dexamethasone cohort; no significant difference was found between the 2 groups ( $p-v a l u e=0.093$ ). The mean satisfaction on a visual analog scale after steroid injection in the methylprednisolone acetate cohort was 3, 0, 0, 0 at 2, 6, 12 and 24-week follow-up; in the dexamethasone cohort it was 4, 0, 0, 0 at 2, 6, 12 and 24-week follow- up. The change in satisfaction on a visual analog scale at 2-week follow-up was significantly different between the methylprednisolone acetate cohort and the dexamethasone cohort ( $p$-value $=0.026$ ), and no significant difference was found between the 2 groups at 6, 12 and 24-week followup.

\section{Tenderness at A1 pulley}

The initial tenderness was 53 in the 2 groups; no significant difference was found between the 2 groups. Tenderness after steroid injection in the methylprednisolone acetate cohort was 29 in 50, 10 in 43, 1 in 41, and 5 in 41 at 2, 6, 12 and 24-week follow-up; in the dexamethasone cohort it was 39 in 48, 21 in 43, 2 in 32, and 3 in 32 at 2, 6, 12 and 24-week follow-up. The change in tenderness of trigger finger at 2 and 6 -week follow-up were significantly different between the methylprednisolone acetate cohort and the dexamethasone cohort ( $p$-value $=0.003,0.025)$, and no significant difference was found between the 2 groups at 12 and 24-week follow-up.

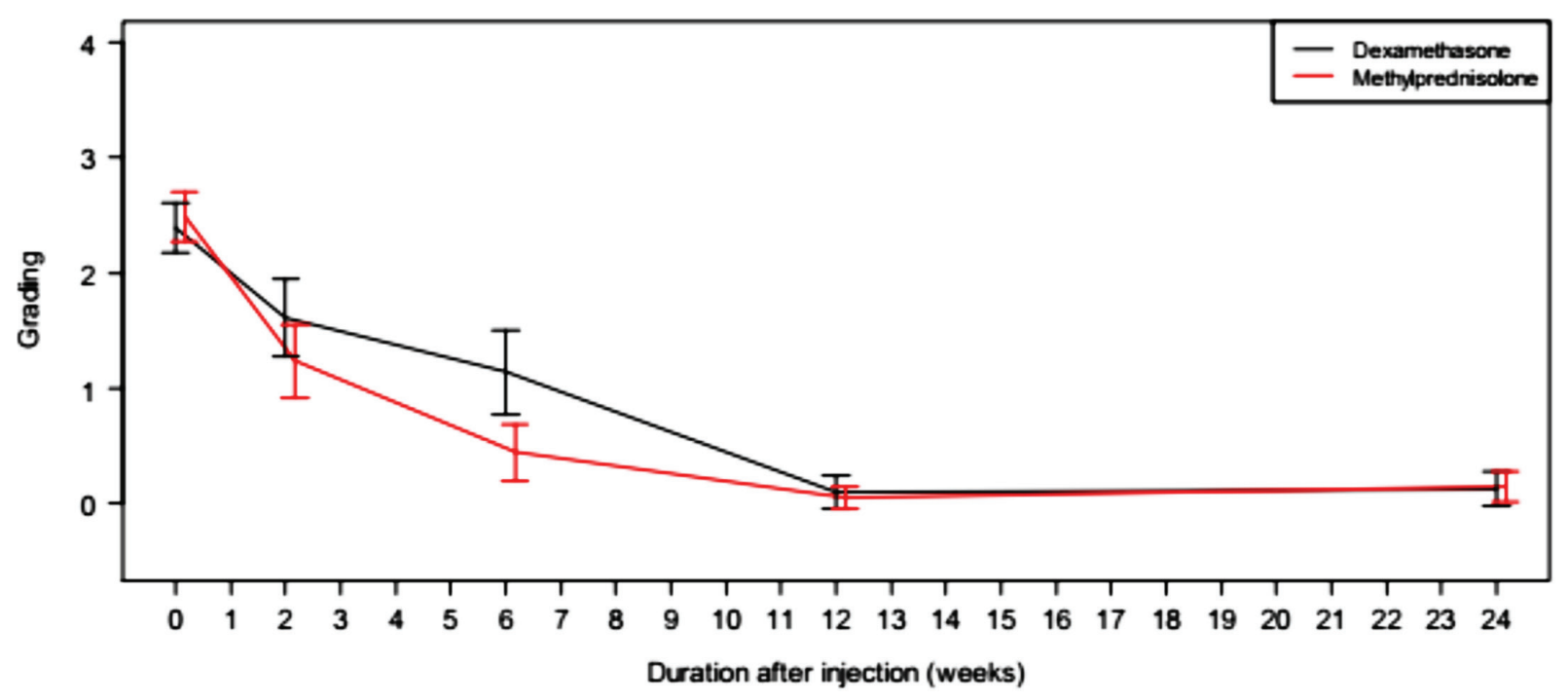

Figure 3 Quinnell grading at 2, 6, 12 and 24-week follow-up 


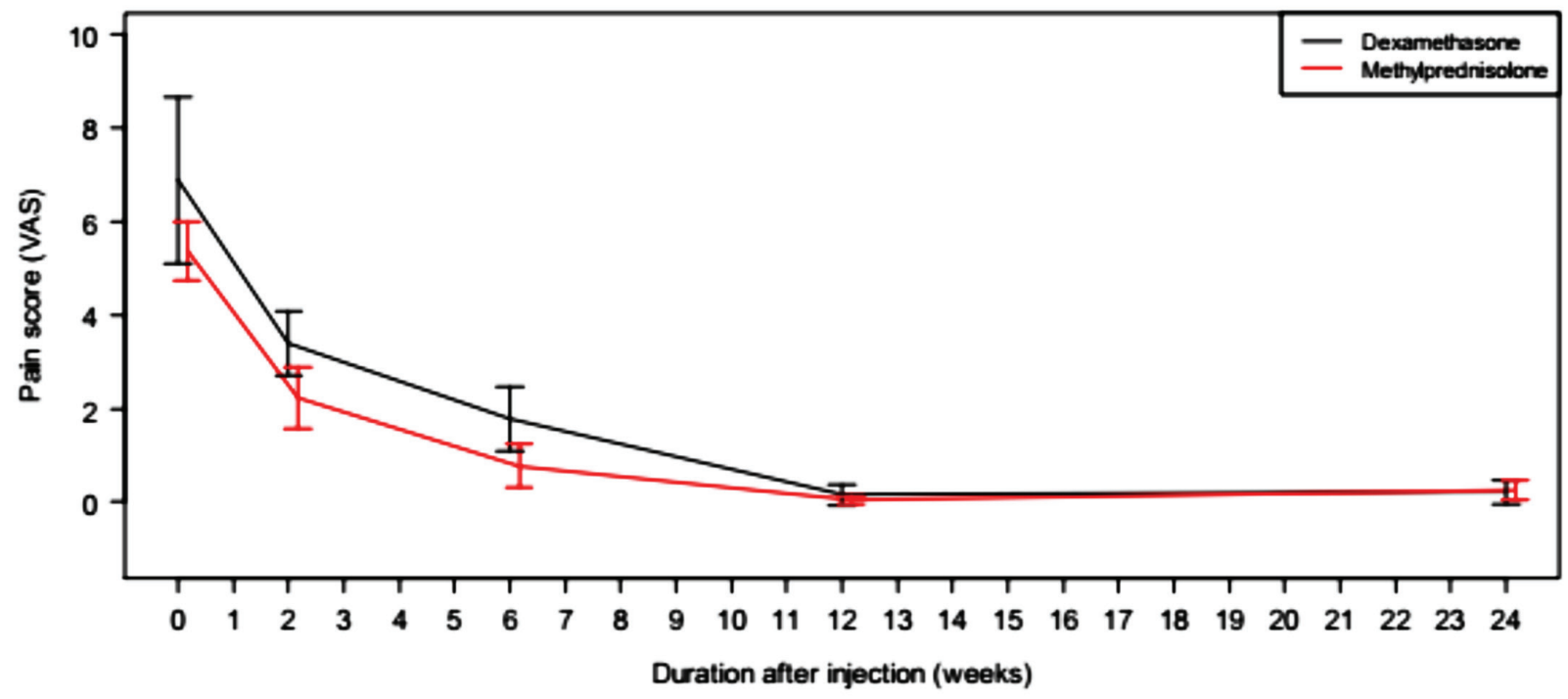

VAS=visual analog scale

Figure 4 The satisfaction on a visual analog scale at initial, 2, 6, 12 and 24-week follow-up

\section{DASH score}

The initial mean of DASH score was 71 and 70 in the methylprednisolone acetate cohort and the dexamethasone cohort; no significant difference was found between the 2 groups. The mean DASH score in the methylprednisolone acetate cohort was 56, 38, 38, and 38 at 2, 6, 12 and 24-week follow-up. The mean DASH score in the dexamethasone cohort was 60,42, 38, and 38 at 2, 6, 12 and 24-week follow-up. The change in grading at 6-week follow-up was significantly different between the methylprednisolone acetate cohort and the dexamethasone cohort $(p-$ value $=0.002)$.

\section{Tip to palm distance $(\mathrm{mm})$}

The initial mean of tip to palm distance was 0 and 0 in the methylprednisolone acetate cohort and the dexamethasone cohort; no significant difference was found between the 2 groups. The mean of tip to palm in the methylprednisolone acetate cohort was 0 at 2, 6, 12 and 24-week follow-up. The mean of tip to palm in the dexamethasone cohort was 0 at 2, 6, 12 and 24-week follow-up. There were no significant differences found between the 2 groups.

\section{Snapping}

The initial snapping was 45 in the methylprednisolone acetate cohort and 46 in the dexamethasone cohort; no significant difference was found between the 2 groups. The snapping after steroid injection in the methylprednisolone acetate cohort was 29 in 50, 8 in 43, 1 in 41, and 1 in 41 at 2, 6, 12 and 24-week follow-up; in the dexamethasone cohort it was 24 in 48, 19 in 43, 1 in 32, and 1 in 32 at 2, 6, 12 and 24-week follow-up. The change in the snapping of trigger finger at 6-week follow-up was significantly different between the methylprednisolone acetate cohort and the dexamethasone cohort ( $p$-value=0.02), and no significant difference was found between the 2 groups at 2, 12 and 24-week follow-up. 


\section{Locking}

The initial locking was 47 in the methylprednisolone acetate cohort and 47 in the dexamethasone cohort; no significant difference was found between the 2 groups. The locking after steroid injection in the methylprednisolone acetate cohort was 4 in 50, 0 in 43,0 in 41 , and 0 in 41 at 2, 6, 12 and 24-week follow-up; in the dexamethasone cohort it was 13 in 48, 7 in 43, 0 in 32, and 0 in 32 at 2, 6, 12 and 24-week follow-up. The change in locking of trigger finger at 2-week follow-up was significantly different between the methylprednisolone acetate cohort and the dexamethasone cohort ( $p$-value=0.023), and no significant difference was found between the 2 groups at 6,12 and 24-week follow-up.

\section{Percutaneous A1 pulley release surgery}

The persistent symptoms of trigger finger after steroid injection at 6-week follow-up was 2 in 11 and 11 in 23 in the methylprednisolone acetate cohort and dexamethasone cohort accordingly. The patients considered percutaneous A1 pulley release surgery after failure of the injection. There was a significant difference between the methylprednisolone acetate cohort and the dexamethasone cohort ( $p-$ value $=0.014)$.

\section{Later recurrence of trigger finger}

The recurrence rate after steroid injection in the methylprednisolone acetate cohort was 1 in 19 at 6-week follow-up and 4 in 40 at 24-week follow-up; in the dexamethasone cohort it was 1 in 30 at 24-week followup. There was no significant difference between the 2 groups.

\section{Side effects}

The stiffness of finger was only one side effect that patients experienced. There was 1 in 43 at 6 -week followup in the methylprednisolone acetate cohort and 1 in 43 at 6-week follow-up in the dexamethasone cohort. There were no serious complications such as infection or tendon rupture.

\section{Discussion}

The corticosteroid injection of trigger finger was initially used as a standard treatment for trigger finger in 1950. The efficacy is vary from $25.0 \%$ to $73.0 \%$. $^{1,10,11}$ In the retrospective study, 90 patients were included, and the overall improvement was 66.0\%, 34.0\% after a first injection, 63.0\% after a second injections, and $66.0 \%$ after a third injections that conformed to this study. ${ }^{7}$ One hundred and six patients enrolled with either methylprednisolone acetate (53 patients) or dexamethasone (53 patients). The overall improvement after methylprednisolone acetate injection was $87.8 \%$, $74.4 \%$ after a single injection and $87.8 \%$ after a second injection. The overall improvement after dexamethasone injection was $90.6 \%, 46.5 \%$ after a single injection and $90.6 \%$ after a second injection. The result supported the hypothesis of this study because the successful treatment of high concentration and high volume of corticosteroid was quite high.

At 6-week follow-up, the change in resolution of trigger finger was significantly different between the methylprednisolone acetate cohort and the dexamethasone cohort $(p-$ value $=0.015)$ by reason of the short onset of action and high concentration of methylprednisolone acetate.

The symptoms of grading, satisfaction on a visual analog scale, tenderness, snapping, locking, the DASH score and tip to palm distance $(\mathrm{mm})$ have been improved in the 2 groups because the corticosteroid can reduce inflammation.

Eleven out of 23 patients in dexamethasone treatment showed remaining symptoms such as positive tenderness and high DASH score, whereas there were only 3 out of 11 patients in methylprednisolone acetate treatment. Most of these patients chose for percutaneous A1 pulley release surgery over continuing corticosteroid treatment. The patients had been advised by the orthopaedist that 
percutaneous A1 pulley release surgery has the high efficacy and low recurrence rate.

In this study, there was no factors to predict the outcome of the treatment and later recurrence. The later recurrence of trigger finger in the methylprednisolone cohort was $10.0 \%$, and in the dexamethasone cohort it was $3.3 \%$; no significant difference was found between the 2 groups. To compare the results of this study to the prospective study which the later recurrence of trigger finger was $23.0 \%$ and can occur at 52-week follow-up. ${ }^{12}$ The minor side effect was found in the study. It was a stiffness of finger. There was 1 in 43 at 6-week follow-up in the methylprednisolone acetate cohort and 1 in 43 at 6-week follow-up in the dexamethasone cohort. The patients with stiffness were sent to rehab in the rehabilitation unit and the clinical symptoms improved. There were no serious complications such as infection or tendon rupture. Fitzgerald et al. $^{13}$, Ring et al. ${ }^{6}$ and Taras et al. ${ }^{5}$ reported that tendon rupture can occur at 56-192 weeks after injection. For this study, if the follow-up time is longer, the later recurrence and the rate of tendon rupture may be found after 24-week follow-up.

In clinical applicable, the orthopaedists should discuss symptom improvement between methylprednisolone acetate and dexamethasone. The methylprednisolone acetate cohort had a better result; only in the short term, but methylprednisolone acetate is more expensive.

The limitation of this study was the short duration of follow-up. The late recurrence and tendon rupture can occur after 24-week follow-up. If the follow-up time is extended more than 24-week, the late recurrence and tendon rupture may occur.

\section{Conclusion}

The methylprednisolone acetate cohort had significantly better satisfaction on a visual analog scale, tenderness, and locking at 2-week follow-up, and had significantly better satisfaction on resolution of triggering, tenderness, snapping, locking and the DASH score at 6-week follow-up compared to those in the dexamethasone cohort.

Although there were no significantly differences in primary and secondary outcomes at 12 and 24-week after injection, the result from our study suggest that both methylprednisolone acetate and dexamethasone had a high efficacy in the resolution of triggering.

\section{Acknowledgement}

We thank Miss Nannapat Pruphetkaew, Department of Epidemiology, Faculty of Medicine, for providing statistical support; and Trevor Pearson for his English proofreading.

\section{Funding sources}

Faculty of Medicine, Prince of Songkla University

\section{Conflict of Interest}

All authors have no conflict of interest.

\section{References}

1. Lambert MA, Morton RJ, Sloan JP. Controlled study of the use of local steroid injection in the treatment of trigger finger and thumb. J Hand Surg Am 1992;17:69-70.

2. Maneerit J, Sriworakun C, Budhraja N, Nagavajara P. Trigger thumb: results of a prospective randomised study of percutaneous release with steroid injection versus steroid injection alone. J Hand Surg Br 2003;28:586-9.

3. Murphy D, Failla JM, Koniuch MP. Steroid versus placebo injection for trigger finger. J Hand Surg Am 1995;20:628-31.

4. Peters-Veluthamaningal C, Winters JC, Groenier KH, Jong BM. Corticosteroid injections effective for trigger finger in adults in general practice: a double-blinded randomised placebo controlled trial. Ann Rheum Dis 2008;67:1262-6.

5. Taras JS, liams GJ, Gibbons M, Culp RW. Flexor pollicis longus rupture in a trigger thumb: a case report. J Hand Surg Am 1995;20:276-7.

6. Ring D, Lozano-Calderon S, Shin R, Bastian P, Mudgal C, 
Jupiter J. A prospective randomized controlled trial of injection of dexamethasone versus triamcinolone for idiopathic trigger finger. J Hand Surg Am 2008;33:514-6.

7. Dala-Ali BM, Nakhdjevani A, Lloyd MA, Schreuder FB. The efficacy of steroid injection in the treatment of trigger finger. Clin Orthop Surg 2012;4:263-8.

8. Sato ES, Gomes Dos Santos JB, Belloti JC, Albertoni WM, Faloppa F. Treatment of trigger finger: randomized clinical trial comparing the methods of corticosteroid injection, percutaneous release and open surgery. Rheumatology (Oxford) 2012;51:93-9.

9. Shakeel H, Ahmad TS. Steroid injection versus NSAID injection for trigger finger: a comparative study of early outcomes. $J$ Hand Surg Am 2012;37:1319-23.
10. Howard LDJ, Pratt DR, Bunnell S. The use of compound F (hydrocortone) in operative and non-operative conditions of the hand. J Bone Joint Surg Am 1953;35-A:994-1002.

11. Kazuki K, Egi T, Okada M, Takaoka K. Clinical outcome of extrasynovial steroid injection for trigger finger. Hand Surg 2006; 11:1-4.

12. Rozental TD, Zurakowski D, Blazar PE. Trigger finger: prognostic indicators of recurrence following corticosteroid injection. J Bone Joint Surg Am 2008;90:1665-72.

13. Fitzgerald BT, Hofmeister EP, Fan RA, Thompson MA. Delayed flexor digitorum superficialis and profundus ruptures in a trigger finger after a steroid injection: a case report. J Hand Surg Am 2005;30:479-82. 